Para enlazar con este artículo / To link to this article:

http://dx.doi.org/10.14198/fem.2019.34.02

Para citar este artículo / To cite this article:

Reid, Martine. «Pour une autre histoire des femmes en littérature». En Feminismo/s, 34 (diciembre 2019): 43-52. Dosier monográfico: Estado actual de la investigación en Literatura francesa y Género: balance y nuevas perspectivas / Etat présent de la recherche en Littérature française et Genre: bilan et nouvelles perspectives, coord. Ángeles Sirvent Ramos, DOI: 10.14198/fem.2019.34.02

\title{
POUR UNE AUTRE HISTOIRE DES FEMMES EN LITTÉRATURE
}

\section{FOR ANOTHER HISTORY OF WOMEN IN LITERATURE}

\author{
MARTINE REID \\ Universidad de Lille, Lille \\ martine.reid@orange.fr
}

\section{Résumé}

Larticle qui va suivre a pour objet la présentation d'un ouvrage collectif, à paraître, qui rassemble, pour la première fois de manière systématique, l'ensemble des informations dont nous disposons à propos de la présence des femmes dans le champ littéraire français, du Moyen Âge au XXI ${ }^{e}$ siècle, avec des considérations sur les littératures francophones. Il contient donc quelques informations et observations générales sur le sujet, et les raisons d'une telle publication, ainsi que quelques observations sur le roman, traditionnellement jugé un genre littérature «féminin».

Mots-clé: femme; féminisme; littérature; histoire littéraire; genre littéraire.

\begin{abstract}
The article consists of a short presentation of a project I directed for several years: for the first time, my colleagues and I will present all the information we have concerning the place, the role, and the works of women in French literature from the Middle Ages to the 21st century, with a chapter dedicated to the Francophone world. The article also includes general views on the subject and the reasons to publish such a book, as well as considerations about the novel, which has been traditionally considered as a «genre» for women.
\end{abstract}

Keywords: Woman; Feminism; Literature; Literary history; Literary genre.

Los contenidos de la revista se publican bajo una licencia de Creative Commons Reconocimiento 4.0 Internacional (CC BY 4.0)

Feminismo/s 34, diciembre 2019, pp. 43-52 
«Il ne faut pas que le passé fasse l'avenir»

(Cixous 37)

Mon propos a pour objectif de présenter le travail collectif que j'ai dirigé et d'illustrer ensuite par l'exemple quelques a priori particulièrement tenaces dans le domaine des femmes en littérature ${ }^{1}$.

\section{FEMMES ET LITTÉRATURE}

Je voudrais présenter pour commencer le travail collectif que mes collègues et moi-même avons élaboré depuis quatre ans et qui sera publié aux éditions Gallimard, dans la collection «Folio inédit», en mars 2020. Il s'agit de la rédaction d'une synthèse sur la présence des femmes dans le champ littéraire français et les œuvres qu'elles ont produites dans cette langue, depuis le moyen-âge jusqu'au XXI ${ }^{\mathrm{e}}$ siècle, à laquelle s'ajoute un chapitre consacré aux francophonies et couvrant essentiellement les deux derniers siècles. Cet ensemble conséquent, qui s'intitule Femmes et littérature. Une histoire culturelle, a réuni une équipe internationale comptant dix spécialistes, avec la participation de Jacqueline Toulet, Eliane Viennot, Joan DeJean et Edwige Keller-Rahbé, Christie McDonald, Florence de Chalonge, Delphine Naudier, Christelle Reggiani, Alison Rice et moi-même.

Quelles ont été nos ambitions? D'identifier d'abord, à la suite de bien d'autres travaux, mais de manière beaucoup plus systématique, l'apport des femmes à la littérature depuis dix siècles, l'une des caractéristiques spécifiques de la littérature de langue française étant la présence continue d'écrivaines depuis le milieu du XII ${ }^{\mathrm{e}}$ siècle. De rappeler ensuite les difficultés considérables rencontrées par celles que l'on a appelées tour à tour auteure (le mot est attesté au XII ${ }^{\text {e }}$ siècle), autrice (utilisé à la Renaissance sur le modèle italien), femmes de lettres (utilisé à l'âge classique avec «auteur»), femmes auteurs

1. Une première version de ce travail a été présentée à l'université d'Alicante le 28 mars 2019, dans le cadre du séminaire sur les femmes en littérature animé par Ángeles Sirvent. Il reprend un certain nombre d'éléments que l'on retrouvera développés dans la préface et dans la partie consacrée au XIX siècle de Femme et littérature. Une histoire culturelle, Martine Reid (dir.), Paris, Gallimard, «Folio essais», 2020.

Feminismo/s 34, diciembre 2019, pp. 43-52 
(qui a la préférence au XIX siècle), auteuses, aut(h)oresses et écrivaines pour être reconnues par la critique, associées aux institutions littéraires, intégrées enfin à un discours portant sur le passé de la littérature. De replacer enfin la production d'œuvres extrêmement nombreuses et diverses dans le contexte littéraire et historique, sociologique, philosophique et anthropologique, étant entendu que, pas plus que leurs contemporains masculins, les œuvres de femmes ne sauraient être confondues entre elles, moins encore être ramenées au seul étalon de leur appartenance sexuée.

Pour ce faire, nous avons utilisé les outils de l'histoire, de l'histoire littéraire, de la sociologie de la littérature (et tout ce qui a trait à la «vie littéraire»), de l'histoire des femmes et de l'histoire des idées, de l'histoire culturelle (notamment l'histoire du livre et de l'édition, de la lecture et de l'éducation), outils auxquels s'ajoutent nécessairement des positions féministes explicites, celles du «genre», voire de son dépassement. La démarche demeure toutefois, globalement, historique, même si nous n'avons pas utilisé les catégories existantes (notamment celles de l'histoire littéraire avec ses genres, ses périodes, ses courants, ses mouvements, etc.) sans les interroger et sans les adapter aux sujets et aux temps qui étaient les nôtres.

Si nous avons souhaité constituer, par siècle, un solide état des lieux, des femmes, et des œuvres, nous l'avons articulé sur une réflexion continue de la différence des sexes et de son fonctionnement spécifique dans le champ littéraire, ainsi que sur les conséquences, partout constatables, de ce que Françoise Héritier appelle «la valence différentielle des sexes» (24). Cette valence, avec une obstination remarquable tout au long des siècles, a dicté des hiérarchies, établi des normes, décidé de traits distinctifs et de règles exclusives; elle a structuré de nombreuses mises en récit de faits littéraires, supposément objectifs, jusqu'à la constitution, à la fin du XIX siècle, d'une histoire nationale et républicaine (ce positionnement idéologique mérite analyse), Histoire de la littérature française de Gustave Lanson (1895) qui a passé très largement les femmes sous silence et les a fait apparaître dans le tableau à titre d'exception.

Au souci de distinction, de hiérarchie, de tenue à distance, notamment dans la vie littéraire, qui s'observe dès le moyen âge répond ainsi une réception critique qui, dans son ensemble, et selon les formes spécifiques qu'elle

Feminismo/s 34, diciembre 2019, pp. 43-52 
peut prendre au fil des siècles, n'en demeure pas moins extrêmement partiale, peu objective, mal documentée, et c'est toujours le cas aujourd'hui.

Existe-t-il pour autant une quelconque spécificité des positions et des œuvres de femmes? Il est extrêmement difficile, à moins de répondre avec le «oui» enthousiaste des féministes des années 1970, d'apporter une réponse simple à cette question, me semble-t-il, sous peine de simplifications abusives, de généralités hasardeuses, de télescopage des époques et d'écrasement des singularités, même si d'évidentes lignes de force, des problématiques, des modes de représentation et de réception se retrouvent et se font écho au fil des siècles. Le discours critique provoque, orchestre, confirme la différence, mais elle a été parfaitement assimilée, introjectée par les deux sexes, et les femmes au premier chef -on peut le constater au XIX ${ }^{\mathrm{e}}$ siècle alors que le nombre d'écrivaines croît assez notablement ${ }^{2}$.

On dira qu'une telle situation n'a rien de très surprenant. Une misogynie tenace a traversé les siècles et a adopté au fil du temps toutes sortes de figures. La littérature en a constitué et en constitue toujours aujourd'hui un excellent terrain d'exercice, ce qui ne peut surprendre quand on se souvient que les notions même d'intelligence et de savoir, de création et de génie ont été -très strictement- réservées au masculin depuis l'Antiquité ${ }^{3}$.

Comment expliquer toutefois qu'après des décennies de féminisme, nous en soyons encore là ? On le sait, la critique et l'histoire littéraire, la «théorie» comme les enseignements traditionnels, sont demeurés en France singulièrement sourds au discours féministe -une telle situation n'était pas celle des pays anglo-saxons où, dès le début des années 60 (le premier département de Women's studies est fondé à l'université Cornell en 1964), les productions féministes se sont constituées en objet de recherche et d'enseignement, et où le fameux canon littéraire a été solidement remis en cause. Les raisons d'une telle surdité sont multiples, et attendent de solides analyses.

Un bref coup d'œil aux ouvrages critiques structuralistes et poststructuralistes permet d'observer toutefois que les grandes figures critiques du temps (toutes masculines) ont conçu leurs modèles théoriques à partir des

2. Sur ce point, voir mes observations dans Des femmes en littérature, introduction.

3. On se reportera sur ce point aux observations de Mary Beard, Femmes et pouvoir (il s'agit à l'origine d'une série de conférences pour la BBC).

Feminismo/s 34, diciembre 2019, pp. 43-52 
grands auteurs du XIX ${ }^{e}$ siècle, et ont notamment fait du «réalisme» et de ses effets le fer de lance de théories promises à une diffusion considérable. Les écrivains servant ainsi les visées de la théorie (Balzac, Flaubert, Maupassant, Zola, Proust) sont ceux que ces critiques (Barthes, Greimas, Todorov, Genette, Hamon, etc.) avaient lus et appréciés dans leur jeunesse, avec lesquels ils avaient été familiarisés à l'école. Curieusement, ils n'ont jamais interrogé les raisons de leur choix, le fonctionnement du champ critique dans lequel leurs propos s'inscrivaient, et ce que ces derniers révélaient de la littérature et de ses grands représentants.

De façon significative, pendant un demi-siècle, les débats n'ont pas porté, en France, sur la nature du corpus de textes à examiner, mais sur la méthode à utiliser pour lire les grands auteurs. C'était déjà une question de méthode qui avait guidé Lanson à l'occasion de ses réformes -ce dernier en effet avait travaillé à remplacer les exercices rhétoriques traditionnels par le commentaire composé et la dissertation, privilégiant ainsi, de son propre aveu, la lecture attentive du texte, et les généralités sur la littérature.

Aujourd'hui, nous sommes les héritiers et héritières d'une telle situation, et d'une méthode (celle de Lanson). La critique, sauf exception, a largement délaissé la théorie. Elle est revenue à ce qu'Alain Vaillant appelle les "piétinements» (15) de l'histoire littéraire ${ }^{4}$ : l'érudition, les éditions savantes et le «biographisme»; il faudrait y ajouter les opérations massives de mises en ligne qui ont précipité une partie de la recherche dans un néo-positivisme dont il reste à penser le sens et à mesurer les effets. Elle est de plus habitée par un clivage tout à fait significatif: d'un côté, il existe des recherches beaucoup plus nombreuses sur les femmes en littérature, parfois sur le «genre» (assez timidement en littérature, pour des raisons institutionnelles), de l'autre des recherches beaucoup plus nombreuses et traditionnelles, parfaitement ignorantes de ces mêmes questions. Les raisonnements tenus, sur les genres littéraires, les courants, les périodes, les manifestations de la vie littéraire prennent très rarement en compte les œuvres de femmes (elles sont inconnues, à de très rares, et très canoniques, exceptions près), pas plus qu'ils ne tiennent en compte l'identité sexuée des auteurs. Ainsi, on ne semble toujours pas sorti(e) s de cette «politique des grands auteurs» constituée à la fin du XIX ${ }^{\mathrm{e}}$ siècle et

4. Cet ouvrage (L'Histoire littéraire) constitue la meilleure synthèse existante sur le sujet.

Feminismo/s 34, diciembre 2019, pp. 43-52 
dont les bornes sont Montaigne et Proust. Une telle situation se retrouve à tous les niveaux de l'enseignement, et, logiquement, dans le «grand public cultivé» qui constitue l'essentiel du lectorat des œuvres classiques.

Cette situation (rapidement) identifiée, il ne s'agit pas de concevoir, de notre côté, un objet fermé sur lui-même, replié sur quelque hypothétique identité féminine et/ou nationale. Nous avons souhaité, à l'inverse, construire un objet hétérogène, ouvert, "global» ${ }^{5}$, forcément incomplet, mais surtout transitoire, en ceci qu'il constitue une étape -nécessaire- pour une reconsidération de l'histoire littéraire dans son ensemble.

Autrement dit, l'histoire que nous avons voulu faire a été conçue comme une partie intégrante d'un tout, qui reste à écrire. Elle va permettre de compléter mais aussi de nuancer considérablement une histoire littéraire à laquelle les femmes appartiennent de plein-droit et dont le récit sur le passé de la littérature ne peut se passer sous peine d'altérer profondément sa réalité, et sa légitimité.

Lhistoire que nous proposons devrait ainsi permettre de se débarrasser des distorsions, des amalgames et des oublis qui caractérisent aujourd'hui encore le discours critique comme l'histoire littéraire; de penser les œuvres des écrivains et écrivaines ensemble, et distinctement, d'en mesurer les échos et les échanges, les convergences et les différences; de procéder, à terme, à une reconsidération générale des œuvres et des réputations, des auteur(e)s majeur(e)s et mineur(e)s, comme du champ littéraire et de son fonctionnement au fil des siècles.

Louvrage n'est qu'un jalon dans l'écriture d'une histoire complète, cette fois, qui n'ignorera pas la présence et la production des femmes dans le domaine de la littérature, qui en fera la matière vivante, multiple d'un seul et même récit. Le passé de la littérature, celui qui fait histoire, et discours de référence, demande d'être appréhendé ainsi, en termes expressément genrés. Cette position suppose un radical changement d'optique, auquel invite le «genre», et qui cassera enfin la vision partielle, partiale, que nous avons tous et toutes de la littérature française depuis Marie de France.

5. C'est le projet, clairement explicité dans la préface, de l'ouvrage dirigé par Christie McDonald et Susan Suleiman. 


\section{LE «GENRE» PAR L'EXEMPLE}

J'en viens maintenant à quelques observations concernant trois points qui, dans l'histoire littéraire, sont liés sans doute possible au féminin. La première concerne l'association entre femmes et romans qui remonte au XVII ${ }^{\mathrm{e}}$ siècle dans la tradition française. Dans son «Essai de l'origine des romans» qui précède le (premier) roman Zayde (1670) de Marie-Madeleine de Lafayette (roman signé par Jean de Segrais, l'un de ses secrétaires), l'auteur, PierreDaniel Huet, autre proche de Lafayette, défend l'idée d'un genre littéraire nouveau avec cette caractéristique singulière: il n'est pas savant (à la différence de la poésie et de la tragédie qui demande des connaissances considérables); «l'ignorance» ne constitue pas un frein à son élaboration. Il cite ensuite quelques exemples (dont les romans de Marie-Madeleine de Scudéry) et reconnaît que les femmes (en réalité une poignée en cette fin de XVII siècle) sont plus habiles dans un tel genre, qu'elles ont contribué de manière magistrale à la constitution de ce genre. C'est incontestablement le cas à l'époque et le nombre de romancières va, pendant quelques années, dépasser le nombre de romanciers, les hommes de lettres du temps ne s'intéressant guère au genre.

Ce qui est intéressant toutefois c'est qu'une telle situation va durer et qu'on retrouve cette association entre femmes et romans tout au long des siècles suivants. Elle semble être ainsi rapidement devenue une véritable assignation générique ${ }^{6}$, en ceci qu'elle va trouver «naturellement recommandée aux femmes, ces dernières déclarant à leur tour -propre de l'injonction efficace- qu'en effet c'est le genre qui leur convient le mieux ${ }^{7}$.

Cette assignation, pour efficace qu'elle soit, est toutefois compliquée de deux manières: les chiffres d'abord, qui rappellent clairement que les femmes ont toujours été minoritaires dans ce domaine; la visibilité ensuite: si quelques romans de femmes ont figuré au nombre des best-sellers dans les premières décennies du siècle (ceux de Sophie Cottin et de Germaine de Staël notamment), la majorité des romans ont été écrits par des hommes, et ce dès le début

6. J'ai proposé le terme dans Des femmes en littérature, chapitre 5.

7. Dans son Lycée, publié en 1797, sorte d'histoire littéraire générale de la littérature depuis l'Antiquité, La Harpe le redit avec force, invitant les femmes à écrire des romans, et dans le domaine qu'elles connaissent le mieux, celui du sentiment.

Feminismo/s 34, diciembre 2019, pp. 43-52 
du XVIII ${ }^{e}$ siècle. Les hommes ont été beaucoup plus nombreux à écrire des romans et à connaître, dans ce genre dit «féminin», des succès considérables (sans jamais néanmoins revenir jamais sur l'idée reçue du roman comme genre féminin).

Les femmes écrivent-elles des romans sentimentaux? C'est la seconde idée reçue qui se voit répétée partout. Manifestement, leur présence de plus en plus visible en littérature à l'extrême fin du XVIII ${ }^{e}$ siècle, succès considérable rencontré par un petit nombre d'entre elles (Riccoboni, Staël ou Cottin) semble avoir renforcé l'idée selon laquelle ce «sous-genre» leur revenait exclusivement. En 1803, Joseph Michaud écrit par exemple dans Le Mercure: «Les femmes semblent s'être exclusivement emparées de ce genre de littérature» (Charles 92), déformant délibérément une situation bien différente, le genre sentimental ${ }^{8}$ étant à la mode pendant un demi-siècle environ et étant pratiqué par des femmes mais surtout par des hommes. Le soi-disant monopole de ces dernières sur cette catégorie de romans -rappelle Shelly Charles- relève en réalité d'une construction critique qui superpose deux lieux communs, le premier liant femmes et sentiment, le second femme et roman.

En réalité, hommes et femmes participent aux romans à la mode et les grand(e)s auteur(e)s n'y échappent pas plus que les auteur(e)s mineur(e)s. Le roman est une forme fourre-tout qui, au fil des siècles, prend toutes sortes d'inflexions (sentimentales mais aussi historiques, philosophiques, exotiques, libertins, gothiques, pédagogiques, «monstres», etc.) qui se confondent et se superposent de multiples façons. Même si les femmes elles-mêmes ont volontiers déclaré qu'elles étaient expertes dans le domaine sentimental, ce sont les hommes qui, au XIX ${ }^{\mathrm{e}}$ siècle sont les «théoriciens» de l'amour, tels Stendhal (De l'amour, 1822), Balzac (Physiologie du mariage, 1829) ou Michelet (LAmour, 1850). Il n'empêche que l'idée reçue continue d'être répétée, même quand la réalité demande sur de telles questions des vues sensiblement plus nuancées.

La troisième idée reçue concerne le lien «naturel» des femmes avec l'idéalisme. Lopposition entre roman sentimental/idéaliste (pour autant que

8. Voir David J. Denby. Sous l'étiquette «roman sentimental» figurent également au début des années 1830 une série de romans dont les femmes n'ont toujours pas l'exclusive, même s'il arrive aux éditeurs de susciter des contributions de femmes sur le sujet: voir Silvia Lorusso. De même Àngels Santa et M. Carme Figuerola (dir.).

Feminismo/s 34, diciembre 2019, pp. 43-52 
ces termes soient synonymes) n'épouse pas la différence des sexes dès la Monarchie de Juillet -et ne peut servir à expliquer, même en partie, l'oubli dans lequel les ouvres de femmes sont tombées ${ }^{9}$. Il me paraît plus juste de lire la mise en place, en littérature, de l'opposition entre idéalisme et réalisme comme un ensemble de glissements successifs s'opérant en réalité sur plusieurs décennies et n'ayant jamais véritablement mis tout le monde d'accord avant le $\mathrm{XX}^{\mathrm{e}}$ siècle.

Une telle opposition s'élabore d'abord à l'occasion du rejet du romantisme, formulée par de jeunes écrivains (Champfleury et Duranty) au cours des années 1840. Ce n'est donc pas une problématique des années 30 mais de la génération suivante. Cette opposition adopte peu à peu une partition sexuée: le romantisme est peu à peu qualifié de féminin, que ses représentants soient des hommes ou des femmes: on trouve des indices chez Goncourt et Barbey, ou encore chez Flaubert quand il critique Lamartine; en 1912, le penseur socialiste Joseph Proudhon qualifie de «femmelins» une série d'auteurs de l'époque romantique ainsi que leur «ancêtre», Rousseau. Toutefois, c'est Emile Zola qui reprend l'argument dans un article qu'il consacre à Balzac et Sand alors que celle-ci vient de mourir. A cette occasion, en 1876, il oppose Balzac, dont il fait le parangon du réalisme, à Sand, dans laquelle il voit la figure de l'idéalisme -pourquoi pas Lamartine ou Hugo? Une telle proposition critique a l'intérêt d'être genrée, de donner à ce qui est valorisé un visage d'homme, à ce qui est dévalorisé un visage de femme, preuve de l'efficacité de cette fameuse «valence différentielle des sexes» (24) bien observée par Françoise Héritier.

L'efficacité de cette distinction ne fait pas l'unanimité tout de suite: Lanson ou Taine, par exemple, condamnent certains textes de Balzac qu'ils jugent bizarres, et ne font pas de Sand le modèle du réalisme. Mais le coup est porté. Zola revient ensuite à plusieurs reprises sur cette opposition entre réalisme masculin et idéalisme féminin, ainsi dans sa «Lettre à la jeunesse», texte dans lequel il n'hésite pas à opposer les «nerfs» à «la virilité du vrai» (103), selon une appréhension «scientifique» (103) des choses, et cette coupure ne se démentira pas.

Dans tous les cas, on le voit, l'association entre femmes et roman, roman sentimental, roman idéaliste est le résultat de constructions critiques qui

9. C'est la thèse de Margaret Cohen.

Feminismo/s 34, diciembre 2019, pp. 43-52 
ont pour particularité de se retrouver encore aujourd'hui dans la plupart des discours tenus sur les femmes et la littérature.

En conclusion à mon propos, il me reste de souhaiter la poursuite de recherches systématiques sur les femmes auteurs, et sans doute plus encore d'inviter à la lecture de leurs œuvres, nombreuses, souvent oubliées, et qu'une grande familiarité de longue date avec les œuvres des auteurs masculins rend parfois difficiles à comprendre, tant nous avons adopté, et avons été invitées à adopter un autre point de vue, d'autres catégories et outils d'analyses pour les penser.

\section{RÉFÉRENCES BIBLIOGRAPHIQUES}

Beard, Mary. Femmes et pouvoir. Paris: Perrin, 2018.

Charles, Shelly. «Le domaine des femmes': roman et écriture féminine dans la critique au tournant des Lumières», Les Femmes dans la critique et l'histoire littéraire. Paris: Belin, 2011.

Cixous, Hélène. Le Rire de la Méduse et autres ironies. Paris: Galilée, 2010.

Cohen, Margaret. The Sentimental Education of the Novel. Princeton: University Press, 1999.

Denby, David J. Sentimental Narrative and the Social Order in France, 1760-1820, Cambridge: Cambridge University Press, 1995.

Héritier, Françoise. Masculin/féminin. La Pensée de la différence. Paris: Odile Jacob, 1996.

Lorusso, Silvia. Matrimonio o morte. Saggio sul romanzo sentimentale francese (1799-1833), Taranto: Lisi Editore, 2005.

McDonald, Christie, et Susan Suleiman, French Global. A New Approach to Literary History, New York, Columbia University Press, 2010 [traduction française: French Global. Une nouvelle perspective sur l'histoire littéraire, Paris: Editions Garnier, 2015].

Reid, Martine. Des femmes en littérature. Paris: Belin, 2010.

Santa, Àngels, et M. Carme Figuerola (dir.). Les romancières sentimentales: nouvelles approches, nouvelles perspectives. Lleida: Edicions de la Universitat de Lleida, col. «Ull Critic», n. ${ }^{\circ}$ 17-18, 2014.

Vaillant, Alain. L'Histoire littéraire. Paris: Armand Colin, 2010.

Zola, Émile. «Lettre à la jeunesse» (1879), Le roman expérimental (éd. Aimé Guedj). Paris: Garnier-Flammarion, 1971. 